\title{
Detection of generalized synchronization using echo state networks
}

D. Ibáñez-Soria, J. Garcia-Ojalvo, A. Soria-Frisch, and G. Ruffini

Citation: Chaos 28, 033118 (2018); doi: 10.1063/1.5010285

View online: https://doi.org/10.1063/1.5010285

View Table of Contents: http://aip.scitation.org/toc/cha/28/3

Published by the American Institute of Physics

\section{Articles you may be interested in}

Using machine learning to replicate chaotic attractors and calculate Lyapunov exponents from data

Chaos: An Interdisciplinary Journal of Nonlinear Science 27, 121102 (2017); 10.1063/1.5010300

Effect of time-delay and dissipative coupling on amplitude death in coupled thermoacoustic oscillators

Chaos: An Interdisciplinary Journal of Nonlinear Science 28, 033119 (2018); 10.1063/1.5009999

Prediction of flow dynamics using point processes

Chaos: An Interdisciplinary Journal of Nonlinear Science 28, 011101 (2018); 10.1063/1.5016219

Phase reduction and synchronization of a network of coupled dynamical elements exhibiting collective oscillations

Chaos: An Interdisciplinary Journal of Nonlinear Science 28, 045103 (2018); 10.1063/1.5009669

Multistability and tipping: From mathematics and physics to climate and brain-Minireview and preface to the focus issue

Chaos: An Interdisciplinary Journal of Nonlinear Science 28, 033501 (2018); 10.1063/1.5027718

Simple and complex chimera states in a nonlinearly coupled oscillatory medium

Chaos: An Interdisciplinary Journal of Nonlinear Science 28, 045101 (2018); 10.1063/1.5011678

\section{PHYSICS TODAY}

\section{ADVANCES IN PRECISION MOTION CONTROL}

Piezo Flexure Mechanisms and Air Bearings

\section{READ NOW}

PRESENTED BY

PI 


\title{
Detection of generalized synchronization using echo state networks
}

\author{
D. Ibáñez-Soria, ${ }^{1, a)}$ J. Garcia-Ojalvo, ${ }^{2}$ A. Soria-Frisch, ${ }^{1}$ and G. Ruffini ${ }^{1,3}$ \\ ${ }^{1}$ Starlab Barcelona S.L., Neuroscience Research Business Unit, Barcelona 08035, Spain \\ ${ }^{2}$ Department of Experimental and Health Sciences, Universitat Pompeu Fabra, Barcelona 08003, Spain \\ ${ }^{3}$ Neuroelectrics Corporation, Cambridge, Massachusetts 020139, USA
}

(Received 24 October 2017; accepted 6 March 2018; published online 27 March 2018)

\begin{abstract}
Generalized synchronization between coupled dynamical systems is a phenomenon of relevance in applications that range from secure communications to physiological modelling. Here, we test the capabilities of reservoir computing and, in particular, echo state networks for the detection of generalized synchronization. A nonlinear dynamical system consisting of two coupled Rössler chaotic attractors is used to generate temporal series consisting of time-locked generalized synchronized sequences interleaved with unsynchronized ones. Correctly tuned, echo state networks are able to efficiently discriminate between unsynchronized and synchronized sequences even in the presence of relatively high levels of noise. Compared to other state-of-the-art techniques of synchronization detection, the online capabilities of the proposed Echo State Network based methodology make it a promising choice for real-time applications aiming to monitor dynamical synchronization changes in continuous signals. Published by AIP Publishing.

https://doi.org/10.1063/1.5010285
\end{abstract}

Synchronization of chaotic systems attracts enormous interest due to its potential applications in digital transmission, secure communications, and physiological modelling. In this paper, we propose a novel machinelearning based approach for the detection of generalized synchronization in temporal series. Two coupled chaotic Rössler attractors have been coupled to construct a series of generalized synchronized sequences interspersed with unsynchronized episodes. We show that these events can be detected by means of recurrent neural networks (RNNs), which present cyclic connections that allow them to process information with a temporal context. Within a reservoir computing (RC) architecture, recurrent neural networks have proved to be able to detect synchronized sequences with excellent accuracy even when noisy environments were simulated. Unlike other synchronization detection methodologies that cannot be applied in a continuous fashion, the approach proposed here exhibits online capabilities that make it an ideal choice to monitor generalized synchronization changes in real time.

\section{INTRODUCTION}

In its everyday use, the concept of synchronization is commonly chosen to imply identical behavior between two interacting systems. However, when the affected systems are chaotic, more sophisticated synchronization forms can exist, such as phase synchronization (where only the phase but not the amplitude of two chaotic oscillations agrees with each other), ${ }^{1}$ lag synchronization (where one of the chaotic systems follows the other with a certain delay), ${ }^{2}$ or generalized synchronization (where the states of the two systems are functionally—but not identically_related in a nontrivial,

\footnotetext{
${ }^{a)}$ E-mail: david.ibanez@starlab.es
}

generally nonlinear manner). ${ }^{3,4}$ Generalized synchronization, in particular, has proven to be a relevant feature in the analysis of neurological disorders such as Alzheimer's disease ${ }^{5}$ and brain tumors. ${ }^{6}$

Over the years, several methods have been proposed for the detection of generalized synchronization, including the replica method, ${ }^{7}$ the synchronization likelihood approach, ${ }^{8}$ and the mutual false nearest neighbor method, ${ }^{4}$ among others. These methods are usually computationally costly, cannot be applied in a continuous manner, and in some cases, suffer from different biases. ${ }^{9}$ Here, we propose a machinelearning-based approach that enables the online detection of generalized synchronization in an effective manner. The method relies on a recurrent neural network to provide the necessary fading memory that allows processing dynamical signals.

Unlike feedforward neural networks, in which a static input-output mapping is applied, recurrent neural networks (RNNs) have cyclic connections that provide memory, implementing a system with dynamical capabilities. ${ }^{10}$ Training RNNs has traditionally been computationally more expensive than training feedforward networks. Echo State Networks ${ }^{11}$ (ESNs) and Liquid State Machines ${ }^{12}$ (LSMs), which were developed independently and simultaneously, and the more recent decorrelation learning rule for RNNs, ${ }^{13}$ are complementary approaches for designing, training and analyzing RNNs within a methodological framework known as Reservoir Computing (RC). $\mathrm{RC}$ is based on the principle that if the network possesses certain algebraic properties, the supervised training of all connections is not necessary. Only supervised training of readout weights is sufficient to obtain an optimal classification performance in many tasks.

Here, we examine the capabilities of ESNs in the task of detecting generalized synchronization changes in synthetic temporal series based on the coupling of two chaotic 
systems. We will use in particular, the well-known Rössler attractors. This paper is structured as follows. In Sec. II, we explain the methodology followed to construct generalized synchronized sequences using coupled chaotic Rössler oscillators. In Sec. III, we provide an overview of the echo state network architecture and their key training parameters. The procedure used to study ESN generalized synchronization detection and results are presented in Sec. IV. We conclude with a discussion in Sec. V.

\section{GENERATION OF IN SILICO TIME SERIES}

The main objective of this work is to explore the capabilities of ESNs for discriminating between generalized synchronized time-series and unsynchronized sequences. In this section, we describe how synchronized chaotic attractors are constructed. To that end, we follow the unidirectional (masterslave) coupling of two Rössler oscillators proposed by Rulkov et $a l^{4}$ A Rössler oscillator is a dynamical system defined by three non-linear ordinary differential equations that exhibit chaotic dynamics. The Rössler oscillator described by the state variables $x$ presented in (1) has been adopted as the driving system, while $y$ in (2) constitutes the coupled response system

$$
\begin{gathered}
\dot{x_{1}}=-\left(x_{2}+x_{3}\right), \\
\dot{x_{2}}=x_{1}+a x_{2}, \\
\dot{x_{3}}=a+x_{3}\left(x_{1}-b\right), \\
\dot{y_{1}}=-\left(y_{2}+y_{3}\right)-g\left(y_{1}-x_{1}\right) \\
\dot{y_{2}}=y_{1}+0.2 y_{2} \\
\dot{y_{3}}=0.2+y_{3}\left(y_{1}-5.7\right) .
\end{gathered}
$$

The manifold $x_{1}=y_{1}, x_{2}=y_{2}$ and $x_{3}=y_{3}$ contains the trajectories of synchronized oscillations. The conditional Lyapunov exponents (CLE) of the response system characterize its asymptotic local stability. ${ }^{14}$ It has been proven that if all CLEs are negative, the response system is stable and shows synchronization. ${ }^{15}$ Reservoir computing has proved its capabilities for the assessment of positive and negative Lyapunov exponents in high dimensional spatiotemporal chaotic systems. ${ }^{16}$

For $\mathrm{a}=0.2$ and $\mathrm{b}=5.7$, Rulkov et $a{ }^{4}{ }^{4}$ proved that trajectories of synchronized motions were stable for a coupling factor of $\mathrm{g}=0.2$, becoming unstable for $\mathrm{g}=0.15$.

Figure 1(a) shows a plot of $x_{2}(t) v s y_{2}(t)$ for $g=0.2$, and Fig. 1(b) for $g=0.15$. In A, we observe a straight line that denotes identical synchronization between driving and response systems, while in $\mathrm{B}$, unsynchronized oscillations appear. By correctly tuning the coupling factor $g$, it is thus possible to force synchronization between the coupled systems. To achieve generalized synchronization, Rulkov proposes the nonlinear transformation of the response system $y(t)$ into $z(t)$ presented in Eq. (3)

$$
\begin{aligned}
& z_{1}=y_{1}, \\
& z_{2}=y_{2}+0.4 y_{3}+0.03 y_{3}^{2}, \\
& z_{3}=y_{3} .
\end{aligned}
$$

Figure $1(\mathrm{c})$ plots $x_{2}(t) v s z_{2}(t)$ for $g=0.2$, where a straight line denoting identical synchronization is no longer observed. Figure $1(\mathrm{~d})$ shows $x_{2}(t) v s z_{2}(t)$ for $g=0.15$. In $\mathrm{C}$, even though a complex relationship between variables is observed, synchronization is not lost since only a non-linear transformation was applied. In this case, $x_{2}(t)$ and $z_{2}(t)$ present generalized synchronization. ${ }^{4}$

Using the behavior reported in Fig. 1, a continuous temporal signal consisting of a series of synchronized sequences interleaved with unsynchronized ones has been constructed. For this purpose, the time-varying square wave coupling function $\mathrm{g}(\mathrm{t})$ is used

$$
g(n)=0.05\left\{\frac{1}{2} \operatorname{sign}\left[\sin \left(\frac{\pi t}{T}\right)\right]+1\right\}+0.15,
$$

where $\mathrm{T}$ defines the up or down state duration in time units. The model has been solved using a 2nd-3rd order RungeKutta method (implemented with the Matlab function ode23) with a fixed integration step of 0.4 time units. The synchronization detection performance is evaluated using a time series of length $10^{6}$ time units and an up/down state duration $\mathrm{T}$ of $10^{5}$ time units. The resultant signal thus concatenates, depending on the initial time, at least 5 generalized synchronized sequences followed by 5 unsynchronized ones.

\section{ESNS FOR GENERALIZED SYNCHRONIZATION DETECTION}

We now discuss the ESN architectures explored and the tests to assess generalized synchronization. Artificial Neural Networks (ANNs) such as the multilayer-perceptron (MLP) presents a feed-forward structure where the information flows from the input nodes, through the hidden nodes and to the output nodes. ${ }^{17}$ Based on the representation theorem, ANNs (and in particular, MLPs) are able to approximate any given arbitrary function. This static input-output architecture makes them suitable for the analysis of stationary problems but, in general, is not adequate to deal with dynamical timedependent problems. Recurrent neural networks (RNNs) incorporate cyclic connections that provide memory capability to the network, and therefore encode time-dependent information. This addition transforms the network into a dynamical system. The network keeps in its internal states non-linear transformations of the input history (fading memory) allowing it to process information with a temporal context. $^{18}$

In this study, RNNs are applied to a supervised binary regression machine learning (ML) problem. In this ML task training, input signals $u(n)$ correspond to the two temporal series whose generalized synchronization wants to be assessed: $x_{2}(n)$ and $z_{2}(n)$, as defined in Sec. II. The network is trained so that its output $y(n)$ is 1 for generalized synchronized samples and -1 for unsynchronized ones. Our RNN, therefore, consists of an input layer of 2 neurons, a hidden layer of $N$ neurons and an output layer of 1 neuron. Input $\left(W^{\text {in }}\right.$ ), hidden $(W)$, and output ( $W^{\text {out }}$ ) connection weights map input signals into output signals, while back-propagation 

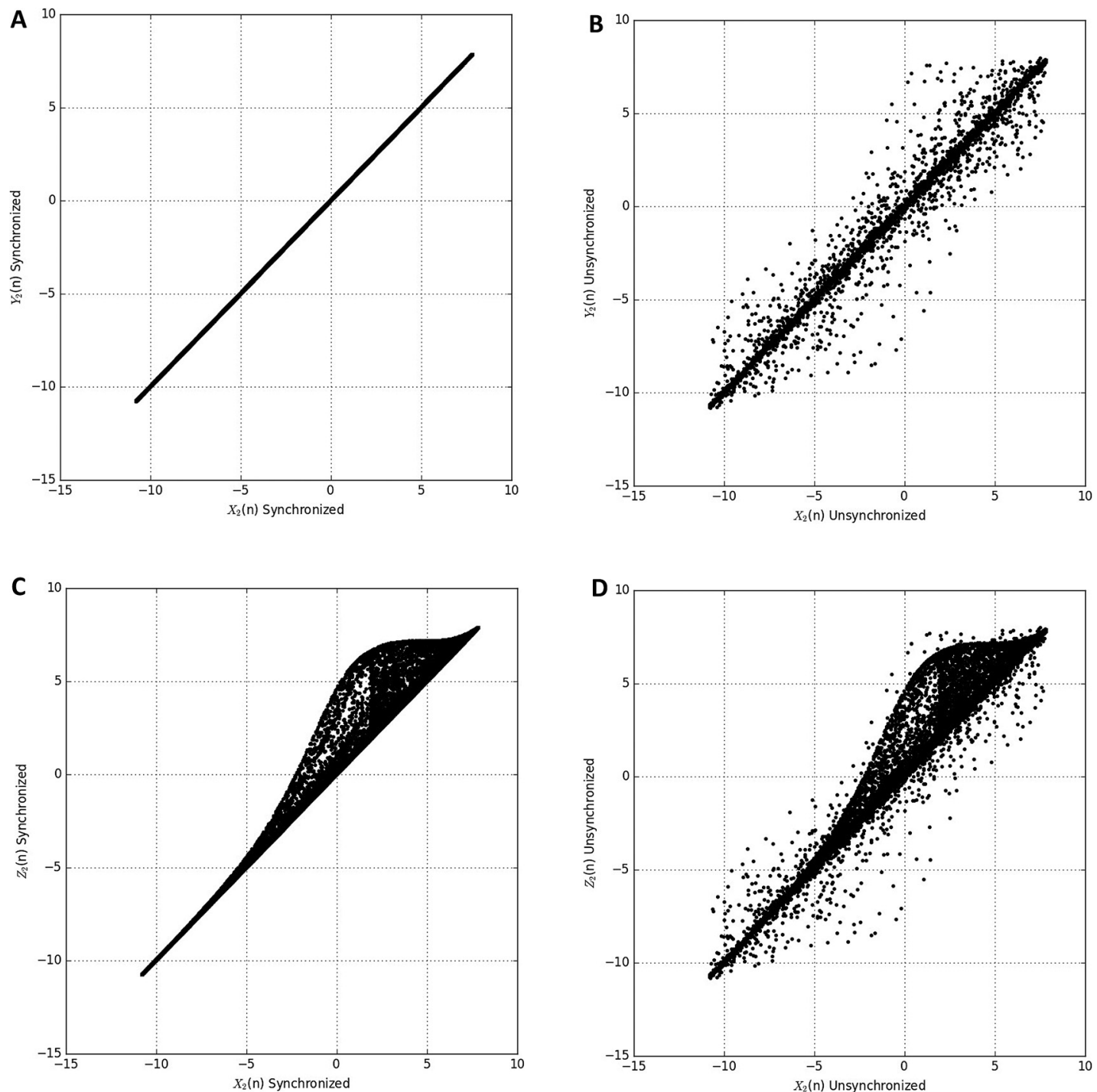

FIG. 1. Dynamics of two coupled Rössler oscillators: (a) $x_{2}(t) v s y_{2}(t)$ for $g=0.2$, (b) $x_{2}(t) v s y_{2}(t)$ for $g=0.15$, (c) $x_{2}(t) v s z_{2}(t)$ for $g=0.2$ and (d) $x_{2}(t) v s z_{2}(t)$ for $g=0.15$.

weights $\left(W^{\text {back }}\right)$ provide memory. The global structure of the RNN is depicted in Fig. 2.

RNN training has traditionally been computationally expensive because of their cyclic nature. ${ }^{22}$ Reservoir Computing (RC), a methodological framework to understand, train, and apply Recurrent Neural Networks (RNNs), was proposed independently and simultaneously with the development of Echo State Networks $(\mathrm{ESNs})^{11}$ and Liquid State Machines (LSMs). ${ }^{19}$ An ESN is a particular form of RNN whose fundamental principle relies on the hypothesis that that if the network presents a certain algebraic property known as the Echo State Property (ESP), only supervised training of readout connections is needed. ${ }^{20}$ The echo state property ensures that the reservoir state does not depend in

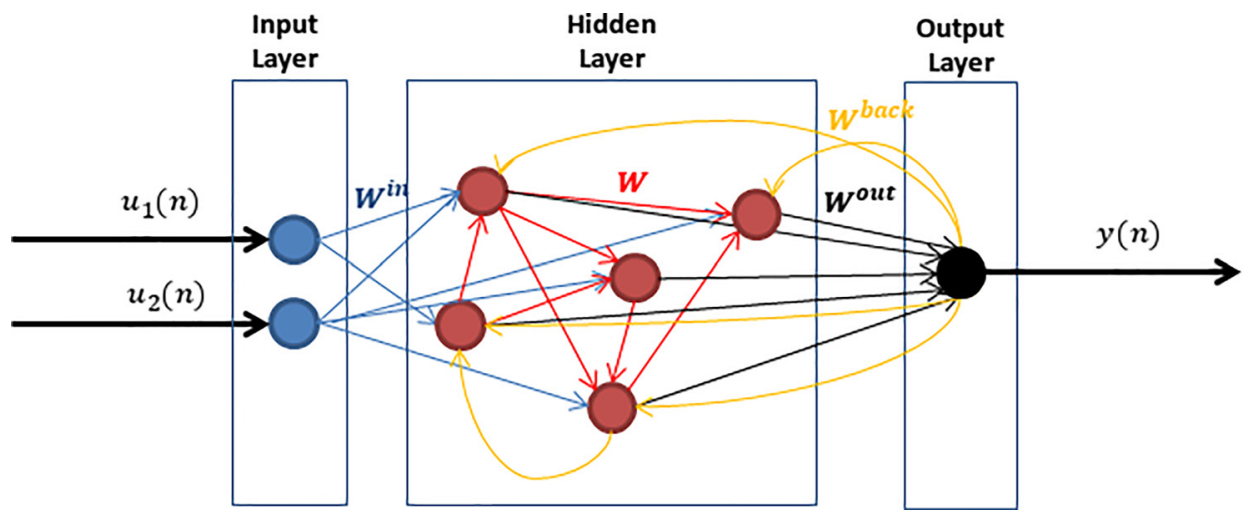

FIG. 2. Proposed Echo State Network model for generalized synchronization detection. 
the long term on the initial conditions, which are thus forgotten as time passes. ${ }^{21}$

The untrained RNN is called the dynamical reservoir (DR) and is formed by the tuple consisting of input, hidden and back-propagation connections. These weight values are fixed and randomly generated according to some parameters, ${ }^{22}$ among which worth pointing out is the so-called spectral radius, calculated as the largest absolute eigenvalue of the internal connection matrix. In practice, if the spectral radius is smaller than one, the echo state property holds for most applications. ${ }^{22}$ Some unlikely exceptions to this rule have been proposed, however. ${ }^{23}$ Additionally, in some situations, the echo state property may also hold for values of the spectral radius larger than one. ${ }^{21}$ In this work, the spectral radius of the DR is always kept below 1.

In machine learning models, good generalization and noise robustness are crucial elements for their application in real-world scenarios. According to ESN training best practices ${ }^{23}$ the most recommended way to learn linear output weights in ESN is ridge regression. Ridge regression regularization removes the need for noise injection in input training signals in order to ensure good generalization, obviates expensive search and has proved to provide high-performing reservoirs. ${ }^{24}$ In our binary regression problem, in which the output is taken to be 1 for generalized synchronized samples and -1 for unsynchronized ones, ridge regression regularization is used to train output weights. The spectral radius, along with the reservoir size and the input scaling, are key global parameters of ESN. ${ }^{22}$ The spectral radius determines the timescale of the reservoir: a small spectral radius induces a faster response, while a larger spectral radius is more suitable for tasks requiring a longer fading memory. The input scaling determines the degree of nonlinearity in the reservoir: while linear tasks require small input scaling factors, tasks with complex dynamics demand larger input scaling values. The reservoir size is determined by the number of internal units. As a general rule, the size of echo state networks is bigger compared to other recurrent neural network approaches. ${ }^{25}$ The number of internal units has to be large enough for the system to learn the complex dynamics associated with generalized synchronization, but not too large, so that the system is generalized well enough, as large reservoirs can lead to overfitting and make the network present poor predictive performance. We perform a grid search (see Sec. IV below) of best input scaling, spectral radius and reservoir size, in order to achieve an optimal ESN parameterization.

The Receiver Operating Characteristic (ROC) curve is commonly used as an effective method to evaluate the performance of binary classification systems. In such two-class prediction problems, outcomes are labeled as positive and negative classes. ROC curves show the trade-off between sensitivity and specificity as a function of a varying decision threshold. In our case, the output test samples corresponding to synchronized sequences are labeled as the positive class and the unsynchronized ones as the negative class. The false positive rate (specificity) and the true positive rate (sensitivity) of the ESN output are calculated for all possible values of the decision threshold. The area under the ROC curve, or simply the Area Under the Curve (AUC), measures the probability of the system of right ranking the positive and negative class samples. An area of 1 means that all samples were correctly classified, while an area of 0.5 represents random classification. AUC is used in this work to evaluate ESN discrimination performance between synchronized and unsynchronized samples.

\section{METHODOLOGY AND RESULTS}

The optimal parameters of the ESN have been determined through exhaustive search in a grid evaluating individually the performance of each tuple under test. Concretely, we used the following value grids: number of internal units - $(10,100,200)$, spectral radius - $(0.01,0.1$, $0.5,0.8,1)$, input scaling - $(0.001,0.01,0.05,0.1,1,5,10$, $100)$ and ridge regularization alpha parameter - $(0.1,1,10)$. The Oger toolbox ${ }^{26}$ has been used to construct the dynamical reservoir, and the scikit-learn toolkit has been used for linear ridge regression regularization.

A Rössler signal formed by a series of 5 generalized synchronized sequences followed by unsynchronized sequences, as described in Sec. II, was used for training. A different signal of same characteristics and the same number of samples but starting at different initial times was used for testing the tuple performance. Hence, we are using for performance evaluation a hold-out validation scheme with 50\% of training samples and $50 \%$ of different test ones. According to the chaotic nature of the Rössler dynamics, by starting at different instants, although the two attractors present a common pattern in the space state, they will develop trajectories that exponentially separate in an unsynchronized manner. ${ }^{27}$ The ESN test output has been smoothed using a 10000 -sample moving average window before performance evaluation.

With the objective of reducing the random effect introduced by the reservoir initialization, each tuple performance evaluation process was repeated 10 times, and its average AUC was used as a performance measure. The best performance achieved a mean AUC of 0.92 with a standard deviation of 0.01 for a spectral radius equal to 0.8 , an input scaling factor of 100 and 200 internal units and unity alpha ridge regression.

Figure 3(a) depicts an example of the output of the outperforming ESN parameter tuple before averaging, which achieves an AUC of 0.58 . The output was scaled in the $[-1,1]$ range for the sake of visualization. The dotted black line represents the aimed ESN output, where 1 represents synchronized sequences and -1 unsynchronized ones. Despite the small AUC obtained, a substantial difference between synchronized and unsynchronized intervals is observed. Desynchronized samples clearly produced an ESN output with a lower higher frequency response. In Fig. 1(b), where the coupled attractors are considered to be unsynchronized, we can observe that many samples lie around the straight line corresponding to synchronization. The ESN discrimination of these sequences appears to be more difficult, as within them not all samples seem to be totally unsynchronized. This behaviour motivates the use of techniques that smooth the ESN output, in order to improve the detection performance. To smooth the ESN 

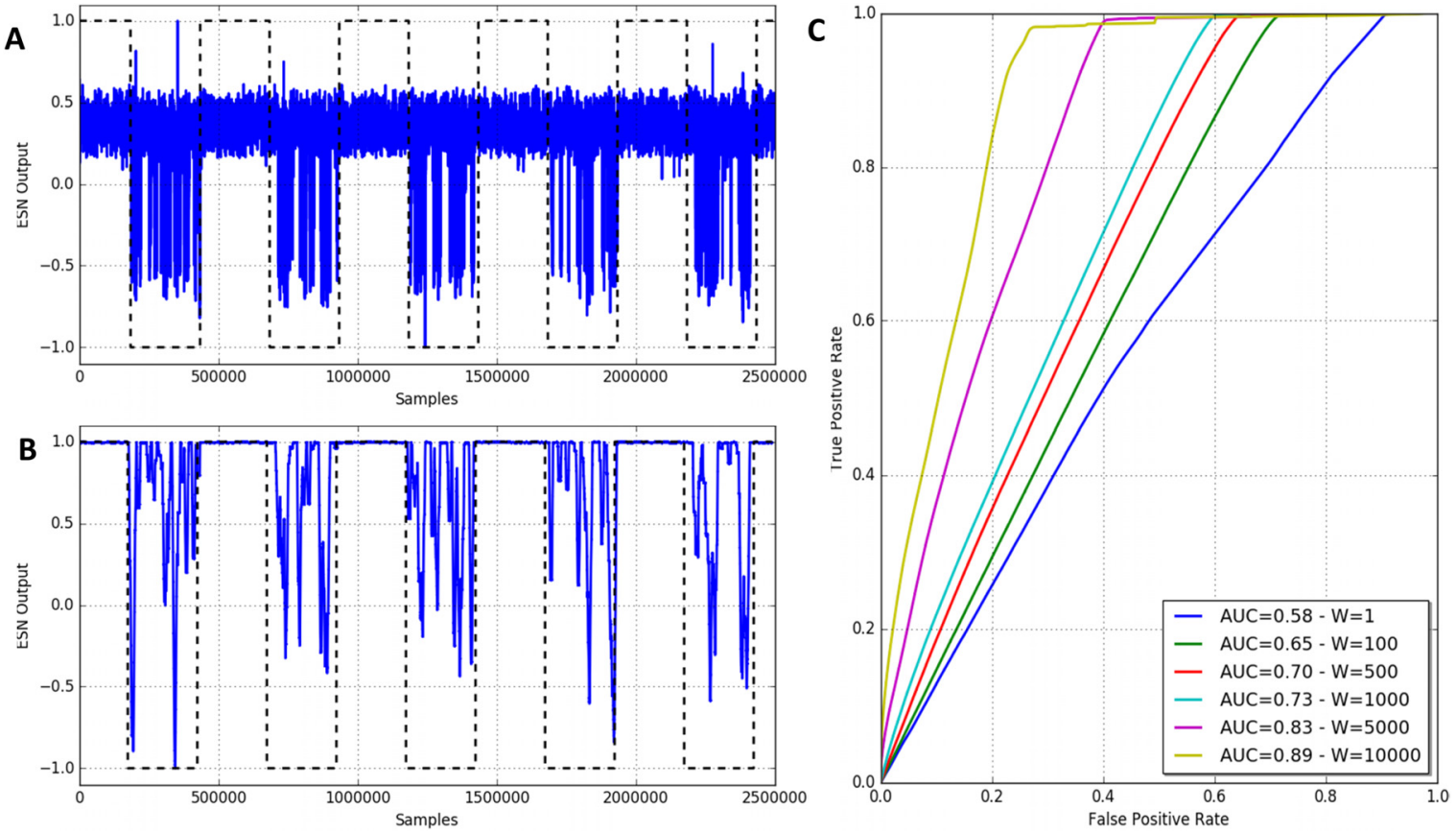

FIG. 3. (a) Unfiltered ESN output for the spectral radius of 0.01, the input scaling of 25, and 500 internal units. (b) ESN output after 10000 samples' moving average for the spectral radius of 0.01 , input scaling of 25 and 500 internal units. (c) Receivers' operator curve calculated for averaging windows of 1,500 , 1000,5000 and 10000 samples.

output, we have used a simple moving average approach implemented as the unweighted mean of the previous $W$ samples, where $W$ stands for the window length in the number of samples. Figure 3(b) shows the ESN output after $W=10000$ samples averaging. In this case, we can observe a better discrimination between generalized synchronized and unsynchronized sequences achieving an AUC of 0.89. Figure 3(c) shows the receivers' operator curve computed for $W=1,100,500$, 1000, 5000 and 10000 samples. As expected, the AUC increases with the averaging window length.

Echo state networks have been proved capable of detecting generalized synchronization in silico time-series. However, in real world scenarios, such as in signals measured by sensors, generalized synchronized signal components may be recorded along with unwanted disturbances and unrelated background activity. In order to simulate realworld scenarios, this nuisance measured activity has been modelled as an additive randomly generated interference in ESN inputs following a normal distribution of zero mean sigma. The noise level is set by multiplying this randomly generated signal by the corresponding noise factor.

$x_{2}(n)$ ESN input as defined in Sec. II is characterized to have a mean of -0.86 , a median of -0.75 and a standard deviation of 4.72 . On the other hand, $z_{2}(n)$ is characterized by a mean of -0.58 a median of -0.55 and a standard deviation of 4.92. Figure 4(a) presents the probability density function (PDF) of both $x_{2}(n)$ and $z_{2}(n)$ that characterizes the distribution of the attractors. The following noise levels have been tested: $0.001,0.01,0.1,1,5$ and 10. Optimal global ESN parameters for each noise level have been determined through exhaustive search in a grid as previously described for 10 iterations and $\mathrm{W}=10000$. Table I displays the largest average AUC obtained for each noise level along with its optimal parameterization. Figure 4(b) displays the average AUC performance with respect to increasing noise levels. As expected, the ESN performance decreases along with the increment of noise; however, ESN networks have proved their robustness in noisy conditions.

\section{DISCUSSION AND CONCLUSIONS}

We have presented a reliable, computationally inexpensive training methodology for the detection of generalized synchronization based on echo state networks. An optimal parameterization of ESNs was able to discriminate between time-locked generalized synchronized sequences from unsynchronized ones delivering an area under the curve of 0.92. An appropriate tuning of ESN global parameters has proved necessary for achieving a good discrimination performance.

The use of ESN in real world applications has been simulated by adding various levels of noise to ESN inputs. Each noise level simulates a different environmental condition. According to the presented results, it has been proved necessary to correctly tune ESN global parameters to each working environment. We could observe a decrease in the input scaling with increased noise levels, but echo state networks prove to be robust to relatively high levels of noise. Given the input signal with approximately zero mean and a standard deviation of 5, we achieved an average AUC of 0.68 with the noise interference simulated as a random signal with zero mean and one standard deviation. This fact shows the feasibility of applying ESN in real world applications. Unlike other generalized synchronization detection methods that cannot be applied in an online fashion, artificial neural networks update their output with every input sample. ESN, therefore, proves to be an ideal choice to develop 

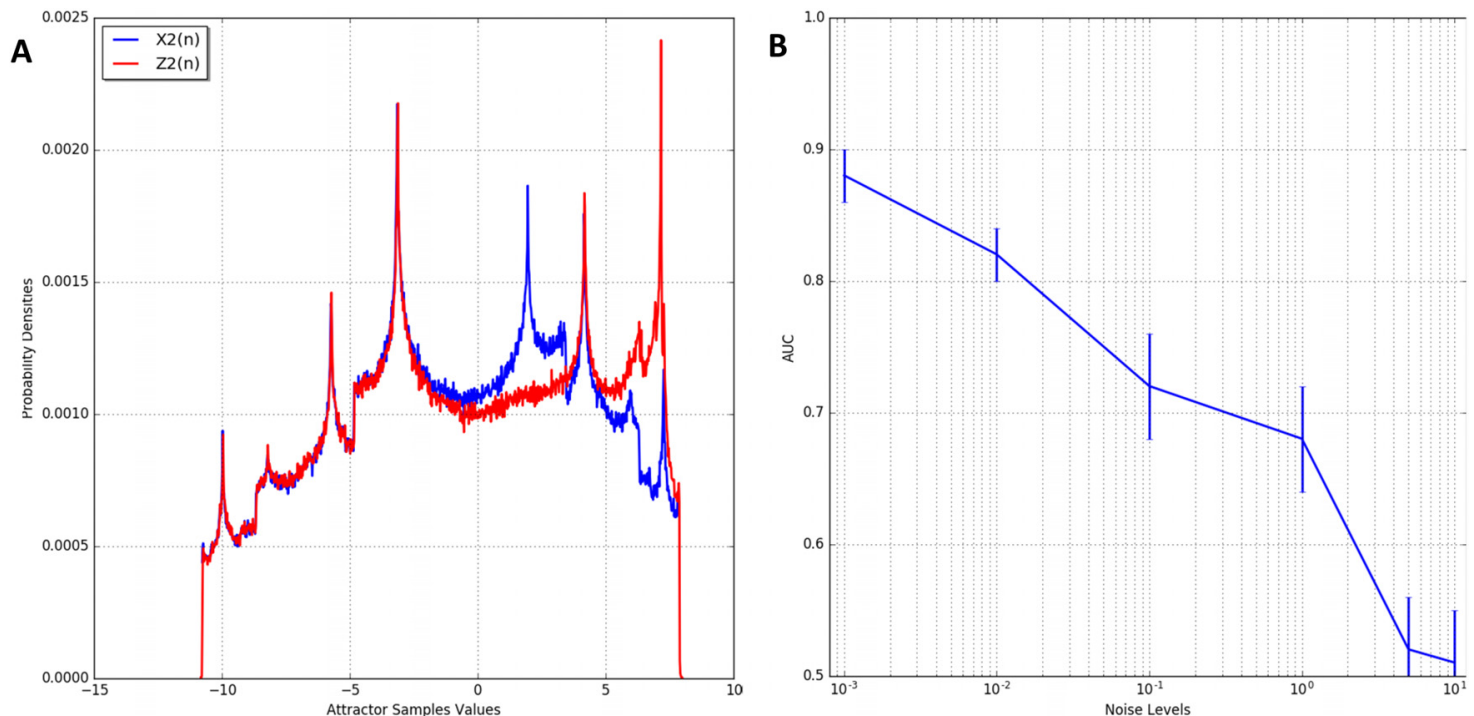

FIG. 4. (a) Probability density function of ESN inputs $x_{2}(n)$ and $z_{2}(n)$. (b) Average AUC performance and standard deviation as a function of increasing additive noise levels.

TABLE I. ESN optimal parameterization and performance for the tested noise levels.

\begin{tabular}{|c|c|c|c|c|c|c|}
\hline Noise level & Avg AUC & Std AUC & Reservoir size & Spectral radius & Input scaling & Ridge alpha \\
\hline 0.001 & 0.88 & 0.02 & 200 & 0.8 & 100 & 1 \\
\hline 0.01 & 0.82 & 0.02 & 200 & 0.5 & 5 & 1 \\
\hline 0.1 & 0.72 & 0.04 & 100 & 0.5 & 0.1 & 0.1 \\
\hline 1 & 0.68 & 0.04 & 100 & 0.5 & 0.1 & 1 \\
\hline 5 & 0.52 & 0.04 & 10 & 0.8 & 1 & 1 \\
\hline 10 & 0.51 & 0.04 & 10 & 0.8 & 10 & 1 \\
\hline
\end{tabular}

applications capable of monitoring generalized synchronization changes in real-time.

The capability of ESNs for the detection of generalized synchronization has been explored by some of us in two real-world scenarios within the field of applied neuroscience, specifically for the analysis of electroencephalographic time series (EEG). In the first work, in the brain-computer interface (BCI) domain, ESNs were used for the detection and characterization of Steady State Visual Evoked Potentials (SSVEPs). ${ }^{28}$ In the second work, ESNs were instrumental in the characterization of abnormal brain patterns in children diagnosed from attention deficit hyperactivity disorder (ADHD). ${ }^{29}$ These two examples can be seen as a demonstration of the applicability of ESNs to real data. All in all, we expect from the theoretical study presented herein to better understand the properties and capabilities of ESN. This can increase the number of applications of this ANN approach for the analysis of temporal series in different scenarios.

\section{ACKNOWLEDGMENTS}

We would like to thank the European Union's Horizon 2020 research and innovation programme who has partially funded the work of David Ibáñez and Aureli Soria-Frisch through the STIPED project under Grant Agreement No. 731827. We would also like to thank the support from ICREA Academia program and the Spanish Ministry of Economy and Competitiveness and FEDER (Project FIS2015-66503-C3-1-P and Maria de Maeztu Programme for Units of Excellence in R\&D, MDM-2014-0370). We also kindly thank the developers of Oger toolbox.

${ }^{1}$ M. G. Rosenblum, A. S. Pikovsky, and J. Kurths, "Phase synchronization of chaotic oscillators," Phys. Rev. Lett. 76(11), 1804 (1996).

${ }^{2}$ M. G. Rosenblum, A. S. Pikovsky, and J. Kurths, "From phase to lag synchronization in coupled chaotic oscillators," Phys. Rev. Lett. 78(22), 4193 (1997).

${ }^{3}$ V. S. Afraimovich, N. N. Verichev, and M. I. Rabinovich, "Stochastic synchronization of oscillations in dissipative systems," Radiofizika 29, 1050-1060 (1986).

${ }^{4}$ N. F. Rulkov, M. M. Sushchik, L. S. Tsimring, and H. D. Abarbanel, "Generalized synchronization of chaos in directionally coupled chaotic systems," Phys. Rev. E 51(2), 980 (1995).

${ }^{5}$ C. J. Stam et al., J. Clin. Neurophysiol. 19(6), 562-574 (2002).

${ }^{6}$ F. Bartolomei et al., Clin. Neurophysiol. 117(9), 2039-2049 (2006).

${ }^{7}$ H. D. I. Abarbanel, N. F. Rulkov, and M. M. Sushchik, Phys. Rev. E 53(5), 4528 (1996).

${ }^{8}$ C. J. Stam and B. W. Van Dijk, Physica D 163(3), 236-251 (2002).

${ }^{9}$ E. Pereda et al., Physica D 148(1), 147-158 (2001).

${ }^{10} \mathrm{D}$. V. Buonomano and W. Maass, "State-dependent computations: Spatiotemporal processing in cortical networks," Nat. Rev. Neurosci. 10(2), 113-125 (2009).

${ }^{11} \mathrm{H}$. Jaeger, "The "echo state" approach to analysing and training recurrent neural networks-with an erratum note," German National Research Center for Information Technology GMD Technical Report, Bonn, Germany (2001), Vol. 148(34), p. 13.

${ }^{12}$ T. Natschläger, W. Maass, and H. Markram, "The "liquid computer": A novel strategy for real-time computing on time series," in Special issue on Foundations of Information Processing of TELEMATIK, 8(LNMCARTICLE-2002-005) (2002), pp. 39-43.

${ }^{13}$ J. J. Steil, "Backpropagation-decorrelation: Online recurrent learning with O (N) complexity," in Proceedings of the 2004 IEEE International Joint Conference on Neural Networks (IEEE, 2004), Vol. 2, pp. 843-848. 
${ }^{14}$ L. M. Pecora and T. L. Carroll, Phys. Rev. Lett. 64, 821 (1990).

${ }^{15} \mathrm{U}$. Parlitz and A. Hornstein, "Detecting generalized synchronization from time series," in Fifth EUROMECH Nonlinear Dynamics Conference ENOC-2005 (2005), pp. 1174-1181.

${ }^{16}$ J. Pathak, Z. Lu, B. R. Hunt, M. Girvan, and E. Ott, "Using machine learning to replicate chaotic attractors and calculate Lyapunov exponents from data," Chaos 27(12), 121102 (2017).

${ }^{17}$ K. Mehrotra, C. Mohan, and S. Ranka, Elements of Artificial Neural Networks (MIT Press, Boston, 1997).

${ }^{18}$ M. Lukoševičius and H. Jaeger, "Reservoir computing approaches to recurrent neural network training," Comput. Sci. Rev. 3(3), 127-149 (2009).

${ }^{19}$ W. Maass, T. Natschläger, and H. Markram, "Real-time computing without stable states: A new framework for neural computation based on perturbations," Neural Comput. 14(11), 2531-2560 (2002).

${ }^{20}$ M. Lukoševičius, H. Jaeger, and B. Schrauwen, "Reservoir computing trends," KI-Künstliche Intelligenz 26(4), 365-371 (2012).

${ }^{21}$ I. B. Yildiz, H. Jaeger, and S. J. Kiebel, "Re-visiting the echo state property," Neural Networks 35, 1-9 (2012).

${ }^{22}$ H. Jaeger, Tutorial on Training Recurrent Neural Networks, Covering $B P P T, R T R L, E K F$ and the "echo state network" Approach (GMDForschungszentrum Informationstechnik, 2002), Vol. 5.
${ }^{23}$ M. Lukoševičius, "A practical guide to applying echo state networks," in Neural Networks: Tricks of the Trade (Springer, Berlin, Heidelberg, 2012), pp. 659-686.

${ }^{24}$ F. Wyffels, B. Schrauwen, and D. Stroobandt, "Stable output feedback in reservoir computing using ridge regression," in International Conference on Artificial Neural Networks (Springer, Berlin, Heidelberg, 2008), pp. 808-817.

${ }^{25}$ F. Triefenbach, A. Jalalvand, B. Schrauwen, and J.-P. Martens, "Phoneme recognition with large hierarchical reservoirs," in Advances in Neural Information Processing Systems Vol. 23 (MIT Press, Cambridge, MA, 2010), pp. 2307-2315.

${ }^{26}$ D. Verstraeten, B. Schrauwen, S. Dieleman, P. Brakel, P. Buteneers, and D. Pecevski, "Oger: Modular learning architectures for large-scale sequential processing," J. Mach. Learn. Res. 13, 2995-2998 (2012).

${ }^{27} \mathrm{C}$. W. Wu, Synchronization in Coupled Chaotic Circuits and Systems (World Scientific, Singapore, 2002), Vol. 41.

${ }^{28}$ D. Ibanez-Soria, A. Soria-Frisch, J. Garcia-Ojalvo, and G. Ruffini, see https://doi.org/10.1101/268581 for Echo State Networks Ensemble for SSVEP Dynamical Online Detection, BioarAxiv (2018).

${ }^{29}$ D. Ibanez-Soria, A. Soria-Frisch, J. Garcia-Ojalvo, J. Picardo, G. GarciaBanda, M. Server, and G. Ruffini, see https://doi.org/10.1101/271858 for Hypoarousal non-stationary ADHD biomarker based on echo-state networks, Bioarxiv (2018). 